\title{
Research Paper: Investigating Validity and Reliability of Early Screening for Autistic Traits-Persian Version (ESAT-PV) in Toddlers
}

\author{
${ }^{*}$ Nahid Vakilizadeh ${ }^{1}$, Ahmad Abedi ${ }^{1}$, Alireza Mohseni Ezhiyeh ${ }^{1}$
}

1- Department of Psychology and Children With Special Needs, Faculty of Education and Psychology, University of Isfahan, Isfahan, Iran.

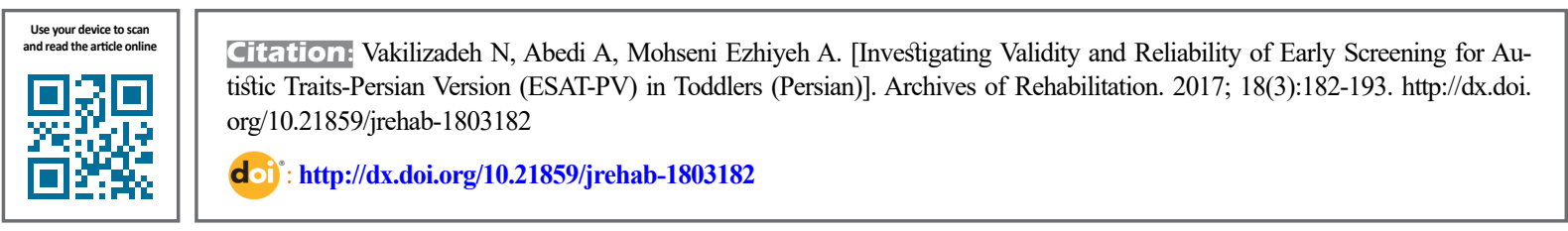

Received: 28 Feb. 2017 Accepted: 20 Jul. 2017

Keywords: Early Screening for Autistic TraitsPersian Version (ESAT-PV), Validity, Reliability

\section{ABSTRACT}

Objective Autism Spectrum Disorder (ASD) is a complex neurodevelopmental disorder that increases in its degree of prevalence on a day-to-day basis. Due to the many problems that the disorder brings to the table for children, families, and society as a whole, its early screening, early diagnosis, and preparing early intervention for this group of children is of extreme relevance. Although many countries have put to use tools designed to screen ASD, Iran has not standardized any tool for this important job. One of the most reliable tools in recent years designed to screen traits of ASD in toddlers is the Early Screening for Autistic Traits (ESAT). Therefore, the aim of the present study is to investigate the validity and reliability of the ESAT-Persian Version (ESAT-PV) among toddlers.

Materials \& Methods This research is a cross-sectional study that was conducted in order to standardize and validate the Persian version of ESAT. The population of the research included all children with ASD in the city of Isfahan. The sample included 72 toddlers selected using the convenience sampling method. After investigating and calculating the Content Validity Ratio (CVR) and Content Validity Index (CVI), like the developers of the main version of the questionnaire did, the mothers participating in the study were asked to answer ESAT-PV questions based on the characteristics of their own children at the age of 14 months. The construct validity was convergent and it was with the Modified Checklist for Autism in Toddlers (M-CHAT) and the Childhood Autism Rating Scale (CARS) and using the Spearman correlation coefficient . Criterion validity was tested by way of comparison of children with and without ASD. To determine the internal consistency of the scale, Cronbach's alpha coefficient was used, and to determine its test-retest reliability, the Intraclass Correlation Coefficient (ICC) was employed. The data were analyzed using SPSS-22.

Results The maximum and minimum of CVER were estimated to be 1 and 0.8 , respectively, and CVI was 0.91 . The correlation coefficient of Modified Checklist for Autism in Toddlers (M-CHAT) and ESAT-PV was 0.56 and that of Childhood Autism Rating Scale (CARS) and ESAT-PV was 0.61. In addition, there was a significant difference between the mean scores of ESAT-PV in the two groups of children with or without ASD ( $t=54.17$ $P=0.001$ ). Cronbach's $\alpha$ coefficient was in the range of 0.73 to 0.77 for questions, and Cronbach's coefficient $\alpha$ for the total scale was 0.76 . Intraclass Correlation Coefficient (ICC) was in the range of 0.93 to 0.77 for questions, and ICC for the total scale was 0.92 .

Conclusion The results of the present study indicate that the ESAT-PV enjoys acceptable validity and reliability and can be applied as a clinical and research instrument for screening autism in toddlers. These results are important because through the investigating and approval psychometric properties of ESAT-PV, operation the concepts of early screening . Thereafter, interested researchers could use ESAT-PV in different groups for identification and screening of ADD. In addition, recommend to rehabilitation experts, psychologists and psychiatrists, with early screening characteristics of autism spectrum disorders in toddlers and acting for the development, implementation and evaluation of the results of early educational and rehabilitation interventions, and thus, be useful in reducing the cost of family and community.

\section{* Corresponding Author:}

Nahid Vakilizadeh, MSc.

Address: Department of Psychology and Children with Special Needs, Faculty of Education and Psychology, University of Isfahan, Isfahan, Iran. Tel: +98 (916) 2388155

E-Mail: nahid.v1991@gmail.com 


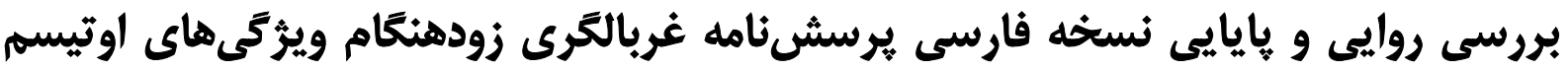 در كودكان نويا
}

\author{
"ناهيد وكيلىزاده'، احمد عابدى'، عليرضا محسنى اريه' \\ ا- كروه روانشناسى وآموزش كودكان بانيازهاى خاص، دانشكده علوم تربيتى وروانشناسى، دانشكاه اصفهان، اصفهان، ايران.
}

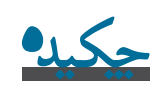

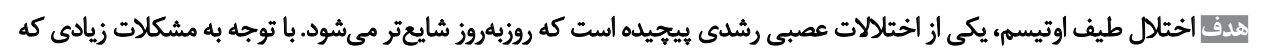

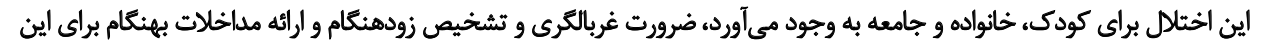

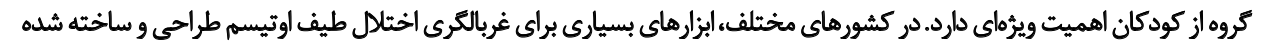

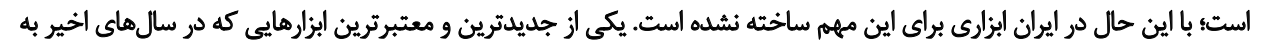

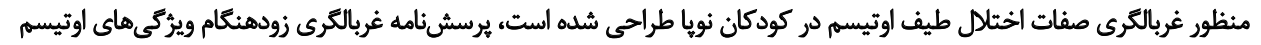
(ESAT)

(ESAT-PV)

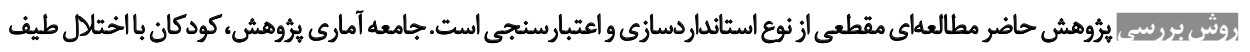

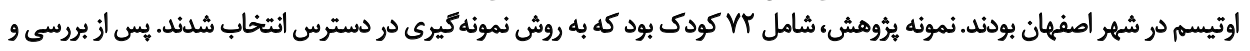

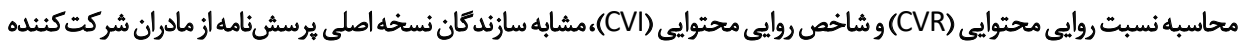

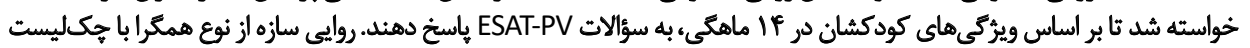

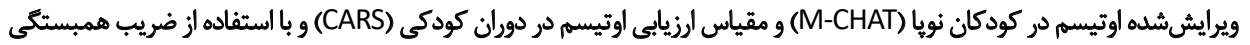

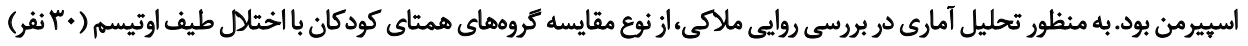

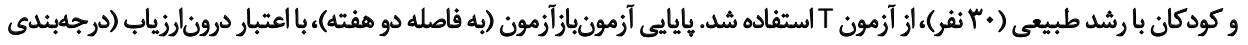

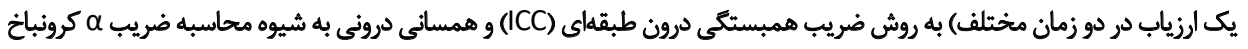

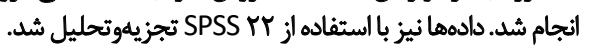

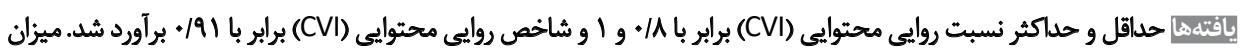

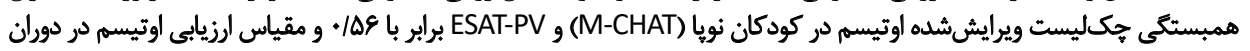

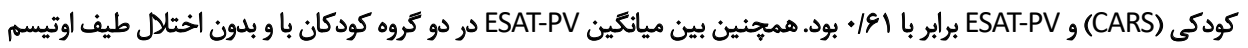

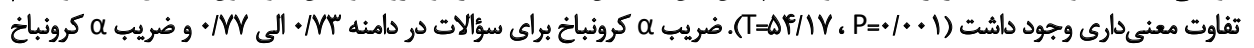

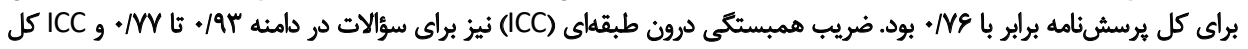

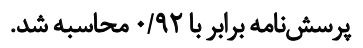

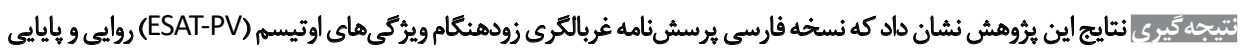

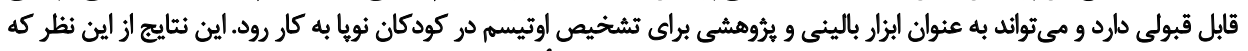

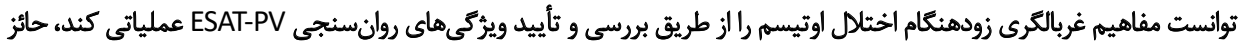

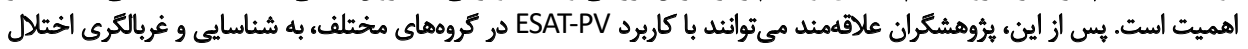

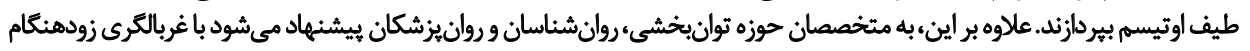

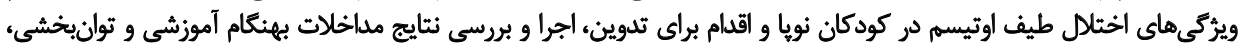

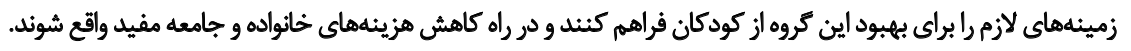

تاريخ دريافت: +1 السفئد هوبات

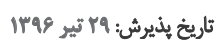


يكى از عوامل اساسى كه موجب تأخير در تشخيص زودهنكًام

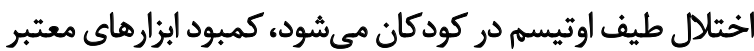

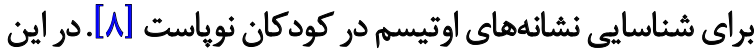

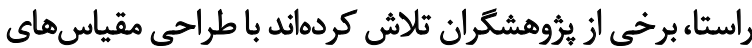

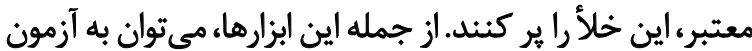

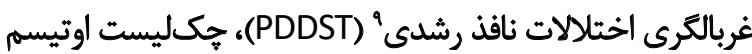

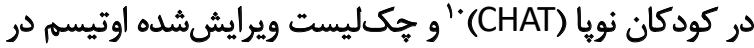

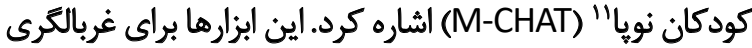

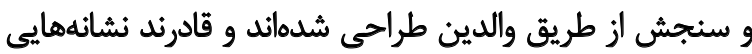

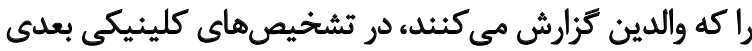

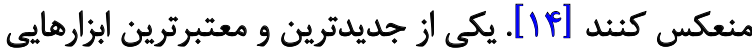

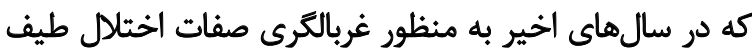

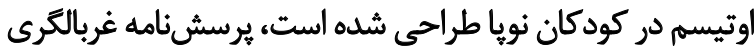

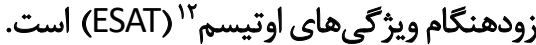

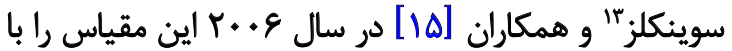

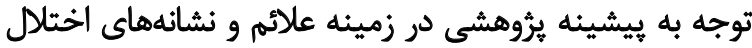

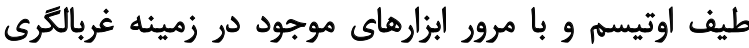

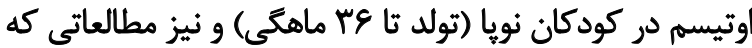

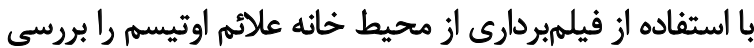

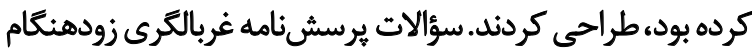

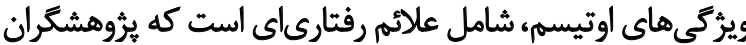

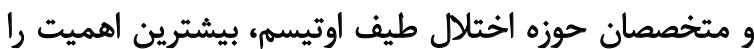

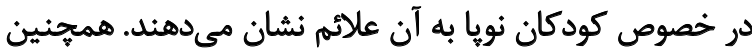

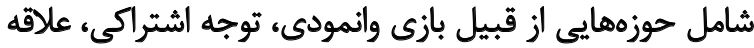

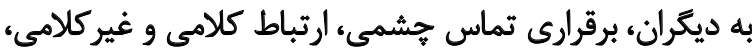

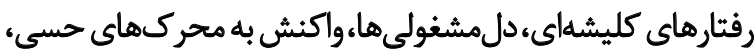
واكنش هيجانى و تعامل اجتماعى است [19إل]

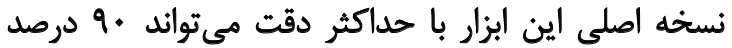

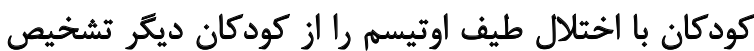

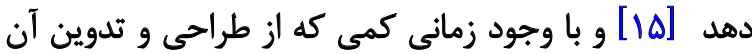

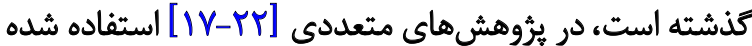

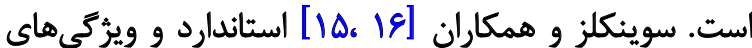

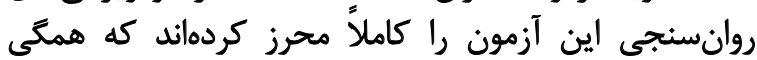

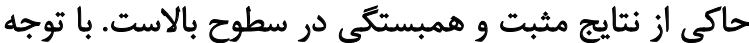

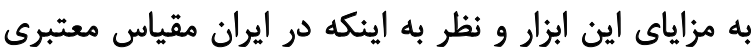

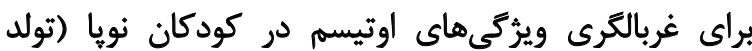

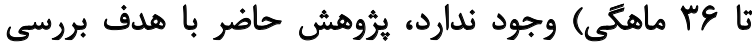

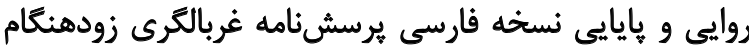

\section{Pervasive Developmental Disorders Screening Test} 10. Checklist of Autism in Toddlers

11. Modified Checklist of Autism in Toddlers

12. Early Screening of Autistic Traits

13. Swinkels

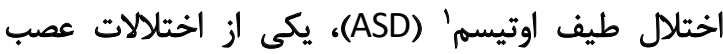

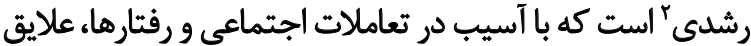

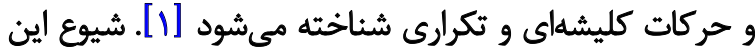

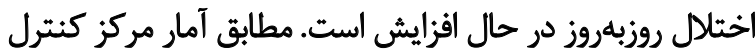

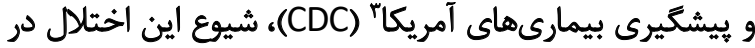

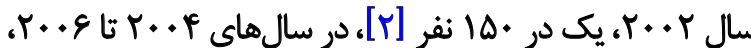

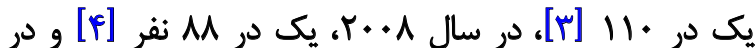

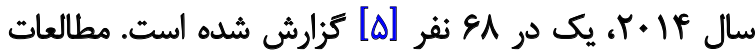

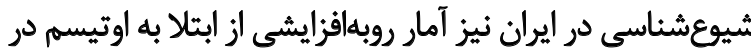

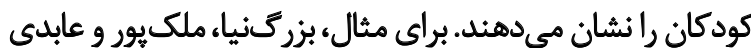

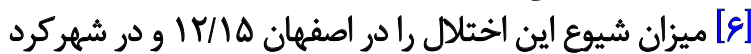

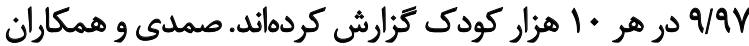

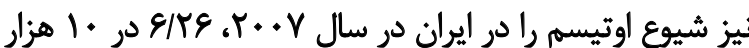

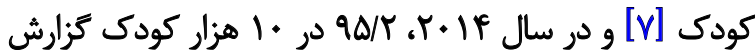

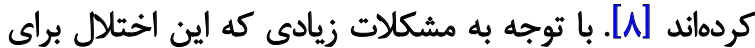

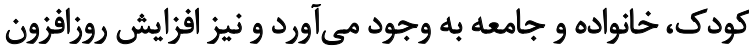

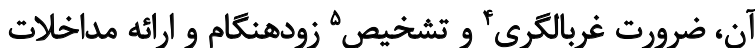

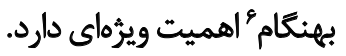

غربالكرى كامى كليدى در شناسايي كودكان مبتلا به

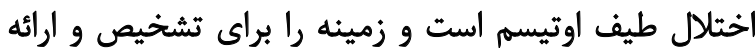

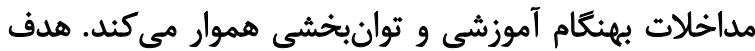

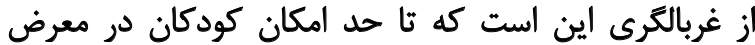

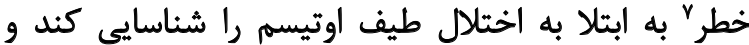

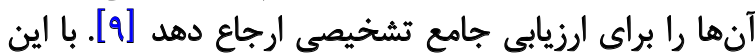

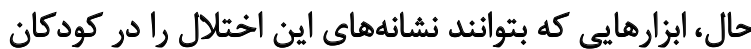

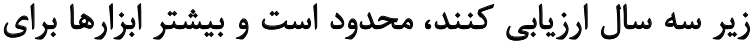

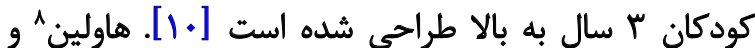

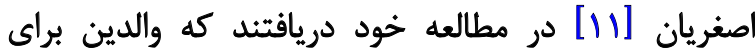

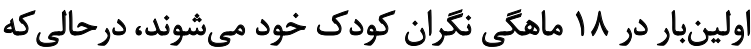

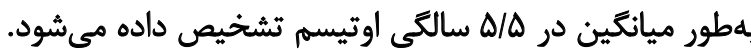

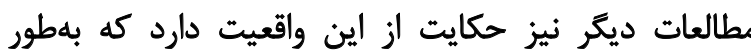

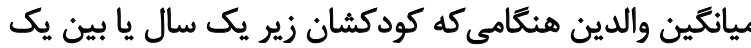

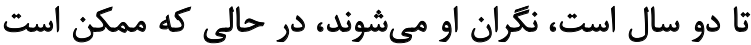

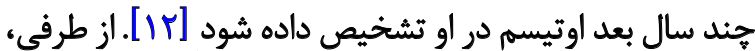

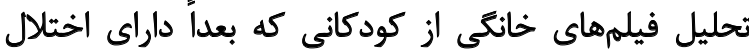

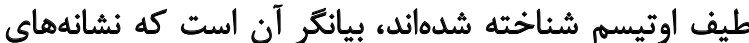

1. Autism Spectrum Disorder

2. Neurodevelopmental Disorder

3. Centers for Disease Control and Prevention

4. Screening

5. Diagnosis

6. Early intervention

7. At risk

8. Howlin 
جكليست ويرايش شده اوتيسم در كودكان نويا (M-CHAT) در سال 999 ا، روبين، فين و بارتن " به منظور ارزيابى كودكان

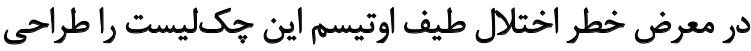

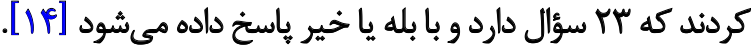

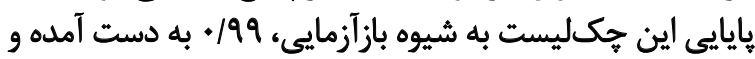

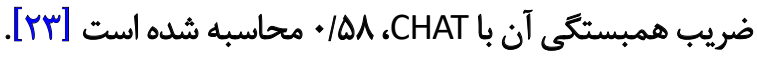

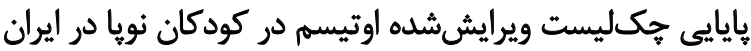

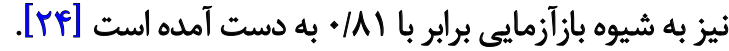

مقياس ارزيابى اوتيسم در دوران كودكى (CARS)

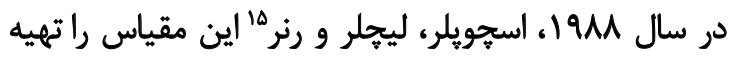

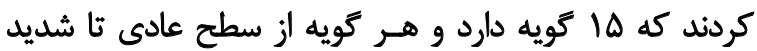

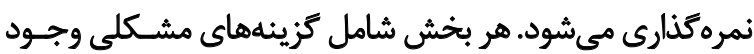

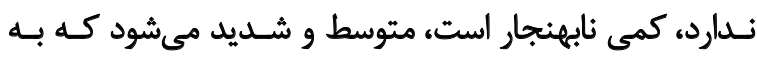

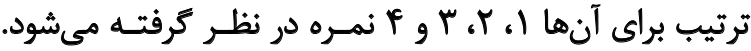

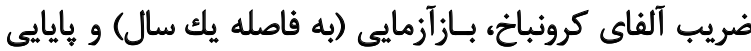

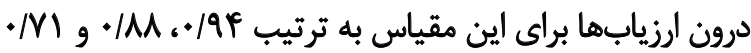

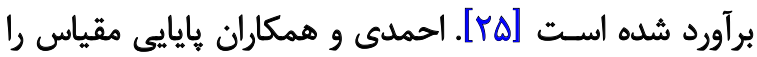

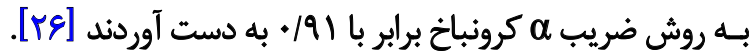

$$
\text { روش الهرl }
$$

با توجه به اينكه در ايران تاكنون مقياس ESAT ترجمه نشده

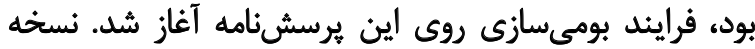

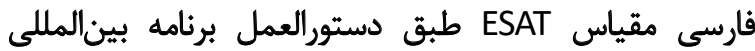

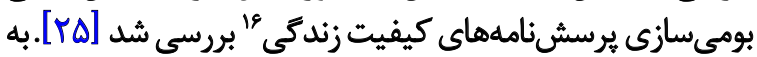

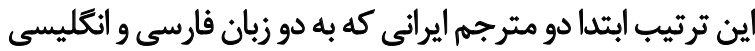

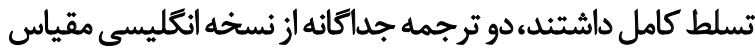

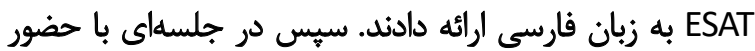

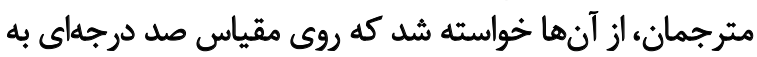

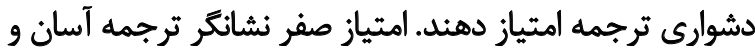

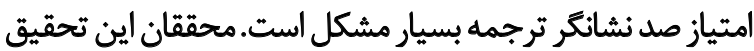

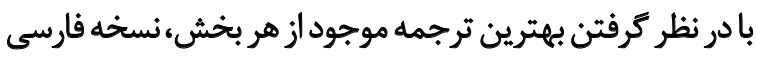

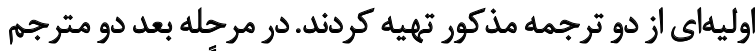

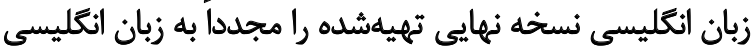

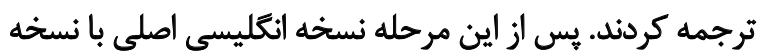

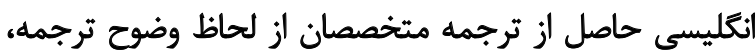

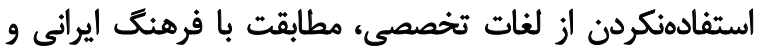

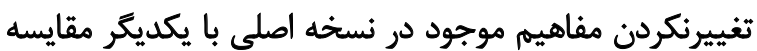

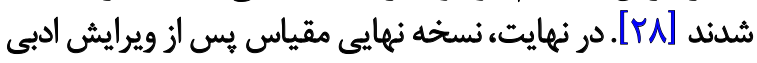
يكى از دانشجويان كارشناسى ارشد زبان و ادبيات فارسي، تهيه نهيه

14. Robins, Fein, \& Barton

15. Schopler, Reichler and Renner

16. International Quality Of Life Assessment (IQOLA)
ويرُكى هاى اوتيسم (ESAT-PV) در كودكان نويا انجام شد.

روش بررسى

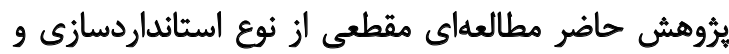

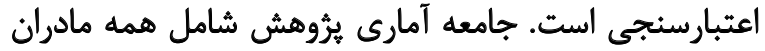

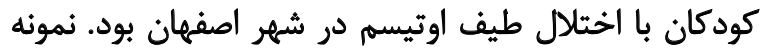

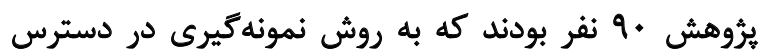

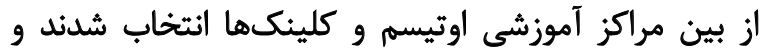

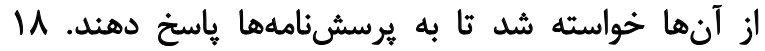

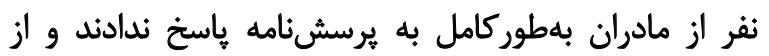

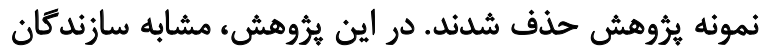

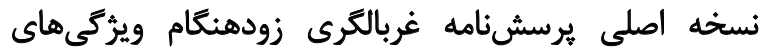

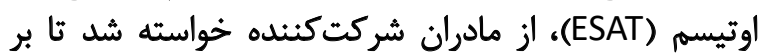

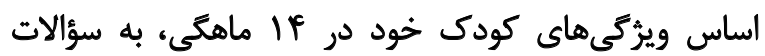

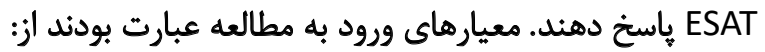

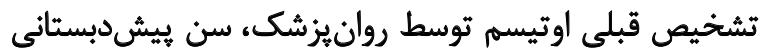

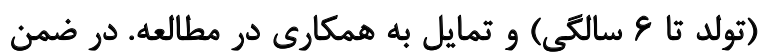

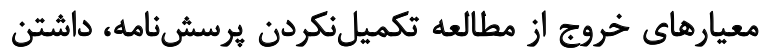

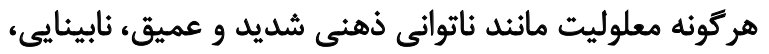

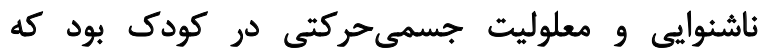

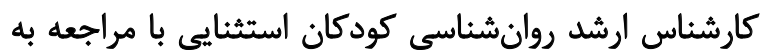

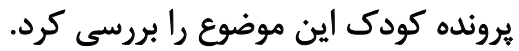

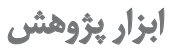

ابزار اصلى يُؤهش حاضر يرسشنامه غربالكرى زودهنيكام

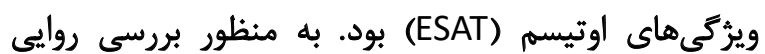

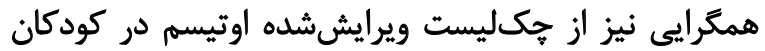
نويا (M-CHAT) و مقياس ارزيابى اوتيسم در دوران كودكى دئي استفاده شد.

\section{برسش نامه غربالكرى زودهنكامويرُكى هاى اوتيسم(ESAT)}

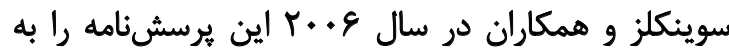

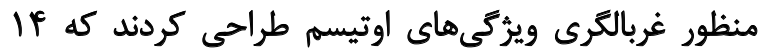

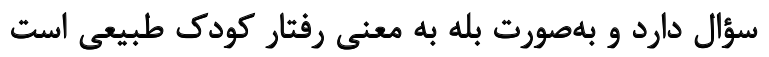

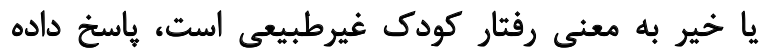

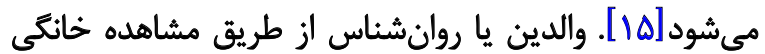

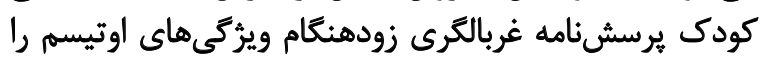

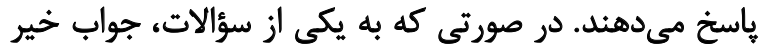

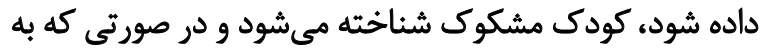

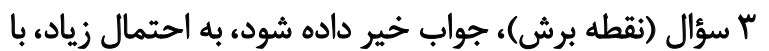

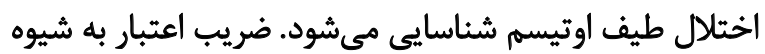

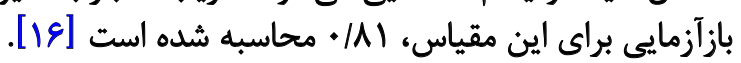




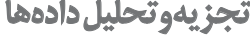

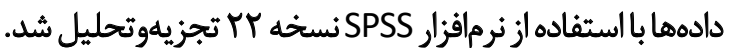

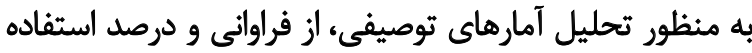

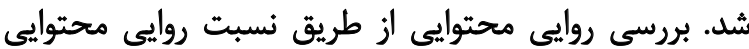

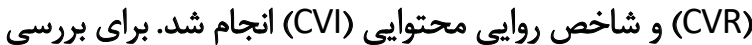

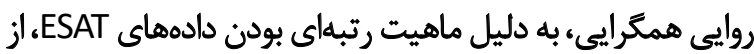

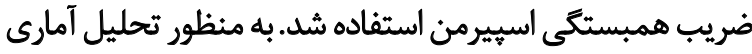

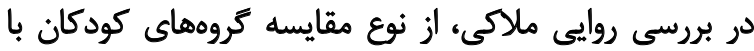

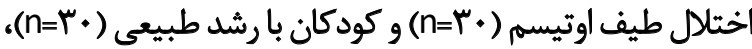

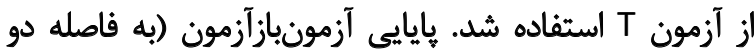

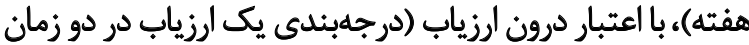

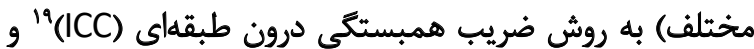
همسانى درونى به شيوه محاسبه ضريب لم كرونباخ انجام شد.

يأقتّههl

ويرُكى هاى كروه نمونه در جدول شماره ا ارائه شده است.

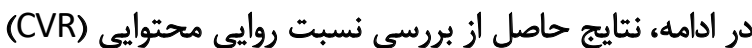

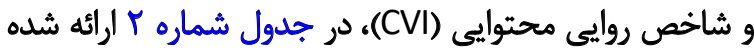

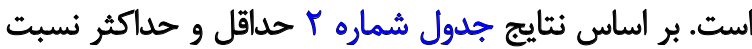

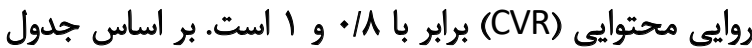

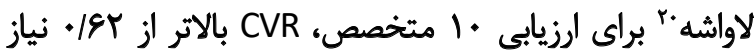

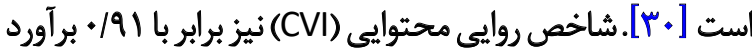

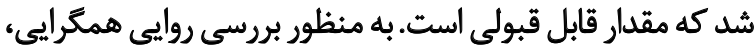
جكليست ويرايششده اوتيسم در كودكان نويا (M-CHAT)

$$
\text { شد (جدول ييوست شماره (). }
$$

براى بررسى نسبت روايى محتوايى" (CVR)، به آراى (CV)

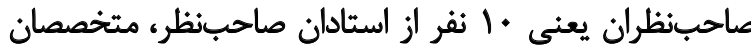

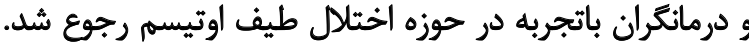

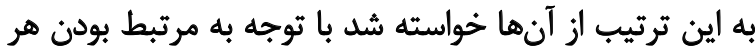

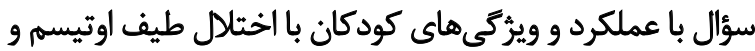

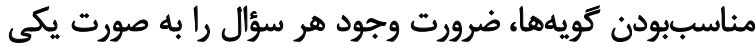

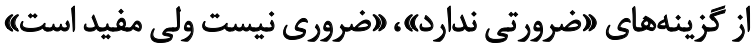

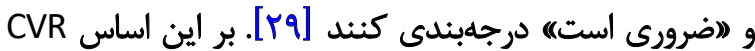

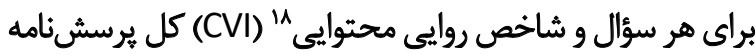

$$
\text { محاسبه شد (جدول شماره r) }
$$

ملاحظات اخلاقي: براى اجراى اين بُروهشه ابتدا هدف از اجراي

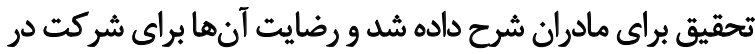

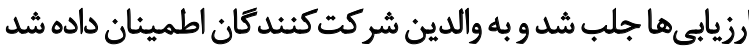

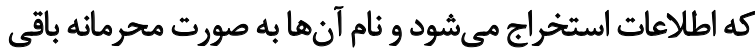

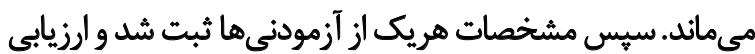
از آنها بهصورت انفرادى انجام شد. ميك.

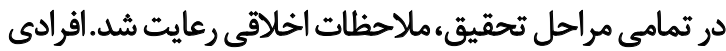

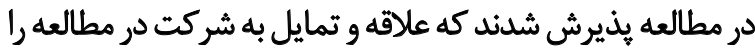

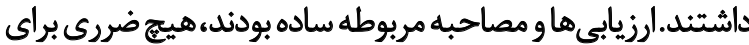

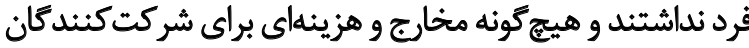

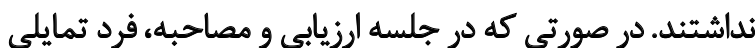
براي ادامه همكارى نداشت، ممانعتى نمى شدئ

17. Content Validity Ratio

18. Content Validity Index

\begin{tabular}{|c|c|c|c|}
\hline درصد & ت تعداد & سطح & متغير \\
\hline \multirow[t]{2}{*}{$1 \mathrm{~W} / 1$} & A & لختر & \multirow{3}{*}{ جنسيت } \\
\hline & & & \\
\hline WA9 & Ex & ل بيسر & \\
\hline e/qf & $\Delta$ & $r$ & \multirow{5}{*}{ سن (سال) } \\
\hline PA/AT & $m$ & $f$ & \\
\hline & & & \\
\hline$\pi / M$ & ra & $\Delta$ & \\
\hline$N / \Delta^{\circ}$ & 9 & 8 & \\
\hline $1 / / 4$ & 1 & بي بوسواد & \multirow{5}{*}{ تحصيلات (مادر) } \\
\hline \multirow[t]{2}{*}{ rV/qf } & $\pi$ & ابتدايع & \\
\hline & & & \\
\hline$\Delta V / / \Lambda$ & $\mathrm{rv}$ & رالهنمايع و دييرستان & \\
\hline $10 / Y A$ & 11 & داتشكاهى & \\
\hline
\end{tabular}

جدول (إويزّى هاى جمعيتشناختى نمونه مطالعهشده (n=rY) 
جدول Y. نمرهماي CVR سؤالات يرسشنامه ESAT

\begin{tabular}{|c|c|c|}
\hline CVR & 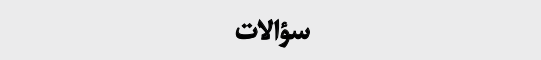 & رديف \\
\hline$\cdot / A$ & علاقه به اسباببازى هاى مختلف & 1 \\
\hline 1 & بازى به شيوهايى مختلف با السباببازىىها & $r$ \\
\hline 1 & آكاهي هيجانى & $r$ \\
\hline.$/ 1$ & واكنش به محركهاي حسى & $\varphi$ \\
\hline 1 & استفاده از حالات جهره & $\Delta$ \\
\hline 1 & برقرارى تماس جشمى & 8 \\
\hline 1 & تاش براى جلب توجه ديكران & $\checkmark$ \\
\hline 1 & حركات بلدنى غيرمتعادل & $\wedge$ \\
\hline.$/ 1$ & نشان دادن و الثاره كردن (به منظور اشتراك علايق باديكران) & 9 \\
\hline$\cdot / 1$ & ع علاقه به ديكران & 1. \\
\hline 1 & علاقه به نوازٔش و در آغوش كرفته شلن & 11 \\
\hline$\cdot / 1$ & ل لبخند به ديكران & ir \\
\hline 1 & علاقه به بازى هاى اجتماعى & ir \\
\hline$\cdot / 1$ & واكنش به صحبتهاي اطرافيان & if \\
\hline.$/ 91$ & CVI امثياز & \\
\hline
\end{tabular}

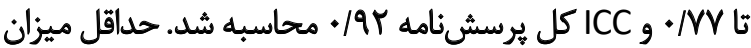

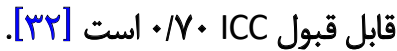

بحث

يُروهش حاضر با هدف بررسى روايى و غايايى نسخه فارسى زئي يرسشنامه غربالكرى زودهنغام ويرَّى هاي اوتيسم (ESAT-PV)

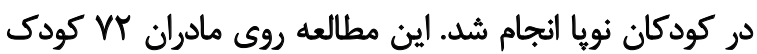

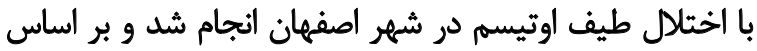

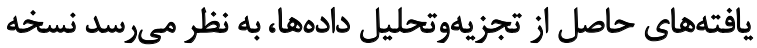

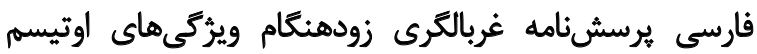
(ESAT-PV)

براي بررسى روايي محتوايى نسخه فارسى يرسش نامه غربالكرى

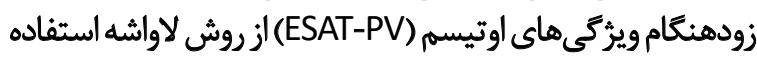

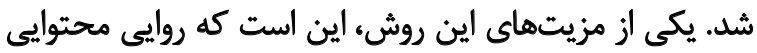

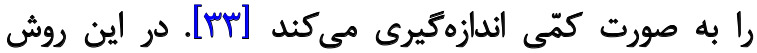

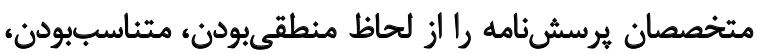

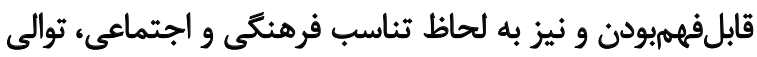

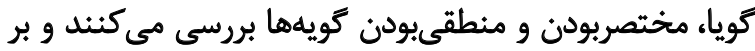

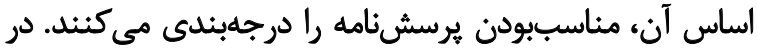

و مقياس ارزيابى اوتيسه در دوران كودكى (CARS) در كنار

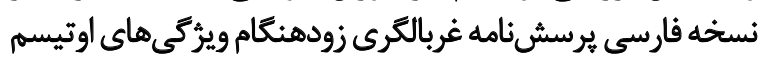

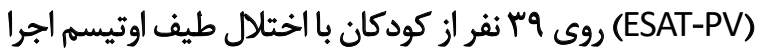

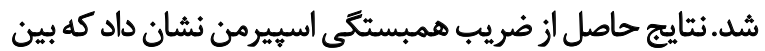

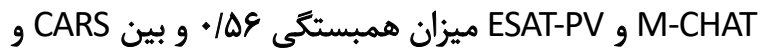
ESAT-PV به منظور بررسى روايى ملاكى، كودكان با اختلال طيف اوتيسم

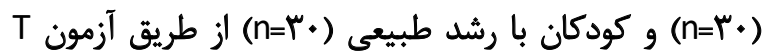

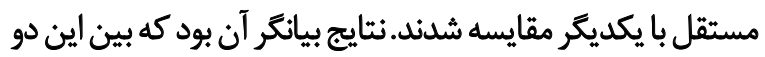

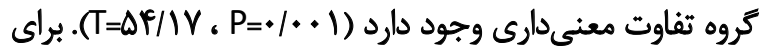

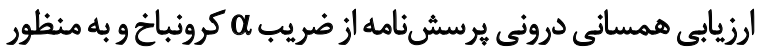

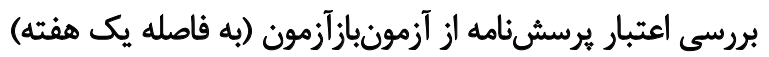

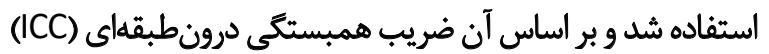

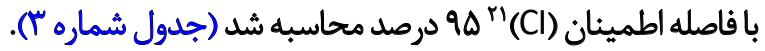

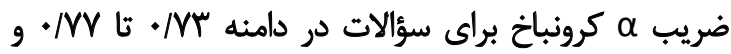

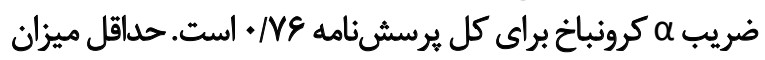

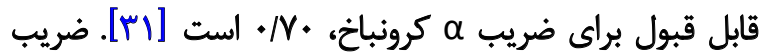

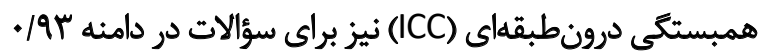

21. Confidence Interval 
جدول با.نتايج بررسى هايايى يرسشنامه ESAT-PV

\begin{tabular}{|c|c|c|c|}
\hline \multicolumn{2}{|c|}{ اعتبار آزمون-باز آزمون (n=ro) } & \multirow{2}{*}{ ضمساني مروني (n=VT) } & \multirow{2}{*}{$\hat{3}$} \\
\hline ICC & فاصله اطمينان \% & & \\
\hline$. / 9 . r$ & $\cdot / 9 \Delta \mid-\cdot / 1 \cdot 9$ & - Na & 1 \\
\hline$+/ N=9$ & $. / 94 \mathrm{~F}-+/ \mathrm{Y}+1$ & $+M$ & $r$ \\
\hline 促 & $. / 91 Y-. / 9 V 8$ & $\cdot M r$ & $r$ \\
\hline$. / 9 . r$ & $\cdot / 9 \Delta \mid-\cdot / 1 \cdot F$ & - Na & $r$ \\
\hline - AMP & $. / 911-. / 8 \Delta$. & $\cdot M$ & $\Delta$ \\
\hline$\cdot / m$ & $\cdot / M Y-. / \triangle Q A$ & - Na & 8 \\
\hline - MEA & $. / 9 M T=. / M T r$ & $\cdot M r$ & $r$ \\
\hline ./9me & $.198 \mathrm{~V}-1 \mathrm{~N}=9$ & - Na & $\wedge$ \\
\hline$\cdot / 2 \wedge 9$ & $. / A q H-. / \Delta K Y$ & - Na & 9 \\
\hline . & $. / N E Q-/ F V \Delta$ & $\cdot M r$ & 1. \\
\hline$+/ V \Delta$ & $+/ A n^{2}-+/ \Delta+\Delta$ & +Na & 11 \\
\hline •/Vq & $\cdot|19 q-/ \Delta 9|$ & $+N G$ & ir \\
\hline.$/ 919$ & 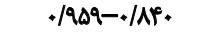 & $\cdot / N F$ & I" \\
\hline - AME & $. / 918=.18 V 8$ & - Na & if \\
\hline.$/ 9 T^{\circ}$ & $. / 909-. / 14 \mid$ &.$N E$ & كل \\
\hline
\end{tabular}

توانبخننى

از طرف ديكر، تكراريذيرى يا قابليت اعتماد نمرات بهدست آمده

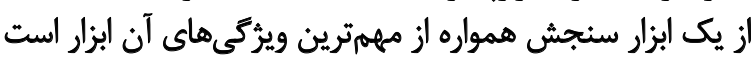

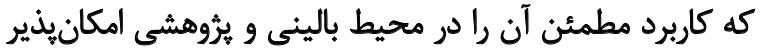

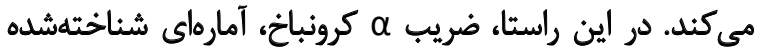

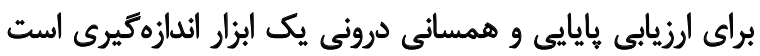

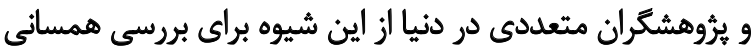

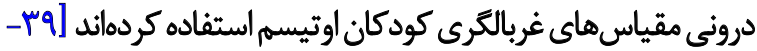

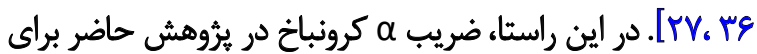
قإبقإل ESAT-PV

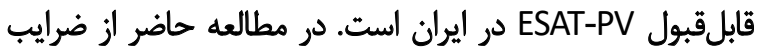

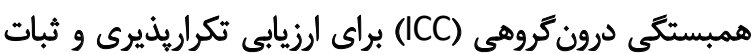

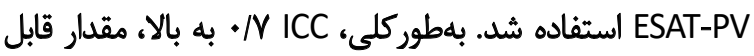

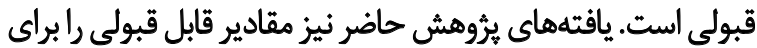

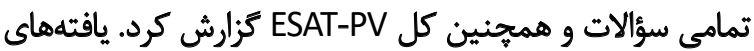

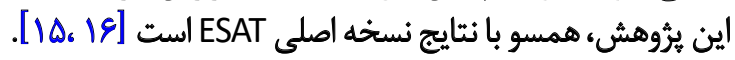
با توجه به نتايج بهدست آمده در اين ثيروهش، متخصصان

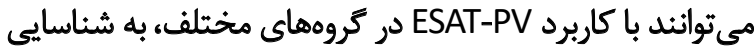

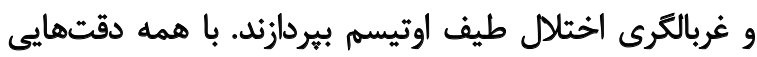
كه ضرورث هر كار علمى است، محدوديتهاى روش الشناختى

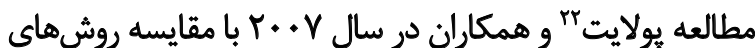

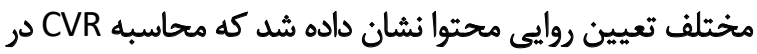
مقايسه با روشهاى موجود ديكر مزاياى بيشترى دارئ دارد. سادئى و

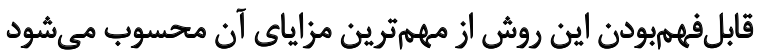

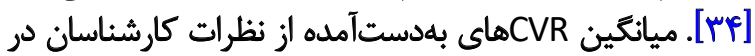

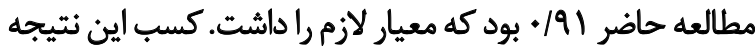
از روايى محتواى مناسب مقياس حاضر است.

همبستخى ESAT-PV با CARS و M-CHAT نشانتر همبستخى

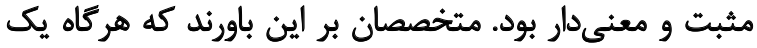

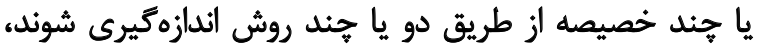

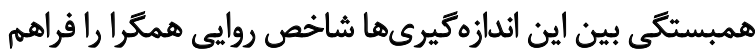

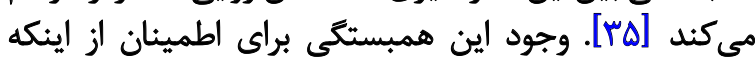

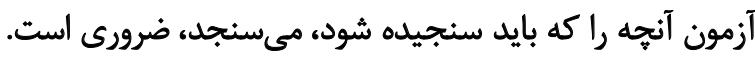

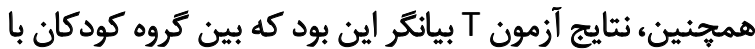

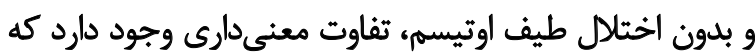

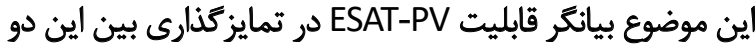

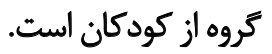

22. Polit 
و تعميمدهى نتايج نمىتواند دور از نظر قرار كيرد. از جمله

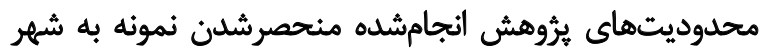

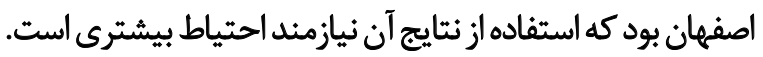

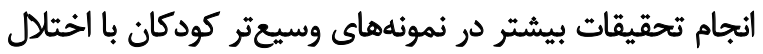

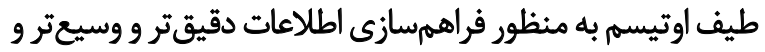

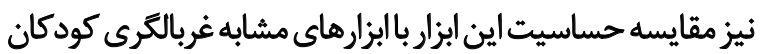

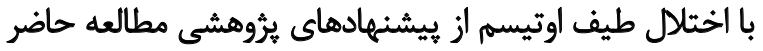

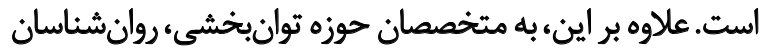

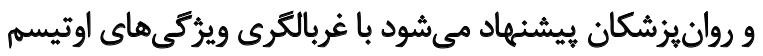

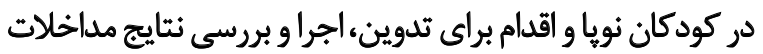

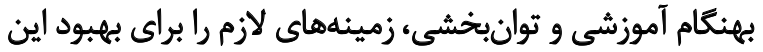

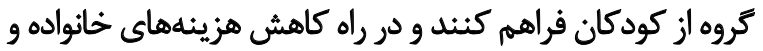

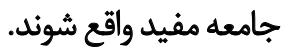

$$
\text { تنيجهابيرى }
$$

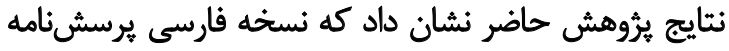

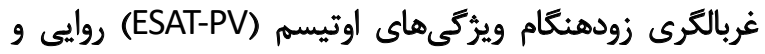

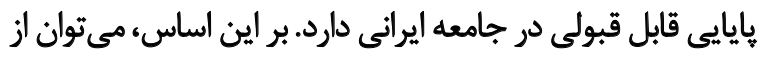

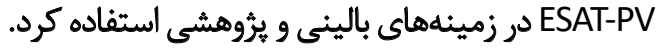

$$
\text { تشكر و قدودانى }
$$

بدينوسيله از تمامى استادان، متخصصان، درمانكران و مادراني

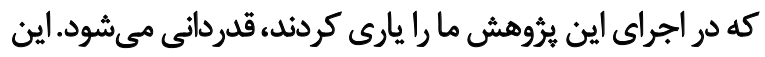
مقاله حامي مالى نداشته است. 


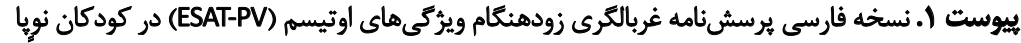

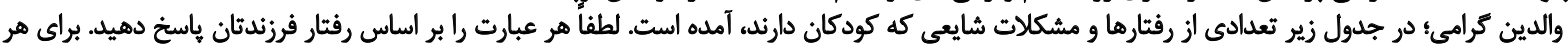

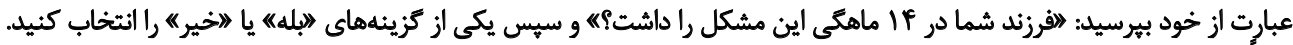

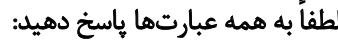

\begin{tabular}{|c|c|c|c|}
\hline خير & بله & 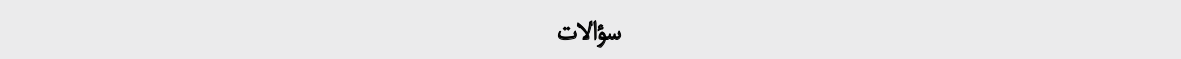 & $\hat{\hat{g}^{2}}$ \\
\hline & & 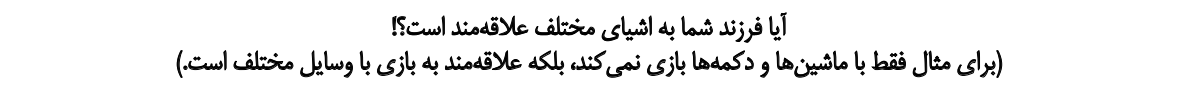 & 1 \\
\hline & & 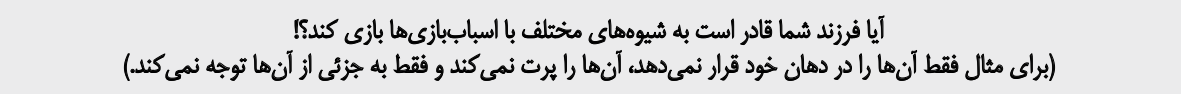 & r \\
\hline & & 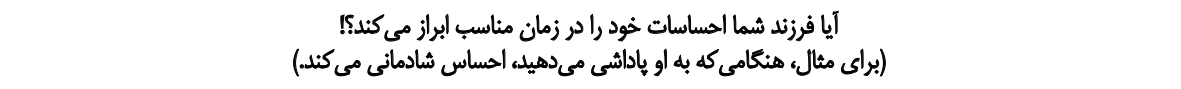 & r \\
\hline & & آيا فرزثد شما به تحريك حسى (ماثثد سرما، كرما، نور، صدا يا درد) به صورت طبيعى عكسلالعمل نشان مىدهـ؟ & f \\
\hline & & 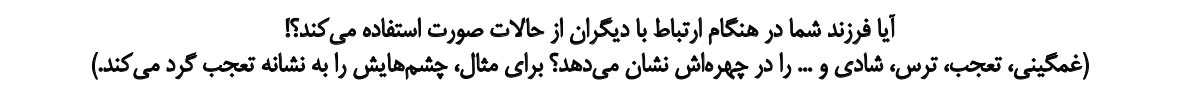 & $\Delta$ \\
\hline & & آيا قادر هستيد بهآسانى با فرزندتان تماس جشمى يرقرار كنيد؟ & $\&$ \\
\hline & & وقتى كه فرزند شما براى مدت اندكى تنها رها مى شوده آيا تالاش مى كند با كريه كردن يا خنديدن، نظر شما را جلب كندي & $r$ \\
\hline & & 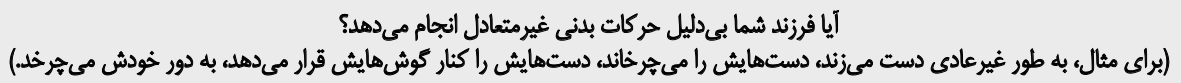 & $A$ \\
\hline & & آيا فرزند شما وسايل مورد علاقهاش را به شما ثشان مىدهد و با انكشت به آنها اشاره هي كند؟ & 9 \\
\hline & & آيا فرزند شما نشان ميدهد كه به ديكر كودكان يا بزركىسالان علاقهمند السث؟ & 1. \\
\hline & & آيا فرزئد شما به نوازش يا در آغوش كرفتن علاقهاي نشان مىدهد؟ & 11 \\
\hline & & آيا فرزندثان تابههال (به منظور برقرارى ارتباط) به شما يا ديكران لبخثد زده است؟ & ir \\
\hline & & 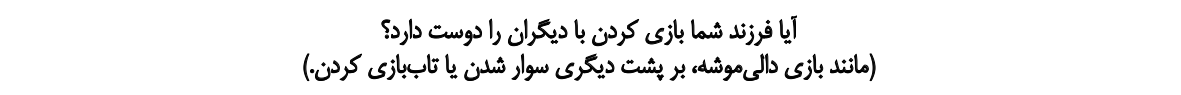 & ir \\
\hline & & آيا فرزند شما هنكامى كه صحبت مى كثيد مثلاً با نكاه كردن، كوش دادن، خثديدن، صحبت كردن يا مِنمن كردن عكس العمل نشان مىدهد؟ & if \\
\hline
\end{tabular}




\section{References}

[1] American Psychiatric Association. Diagnostic and statistical manual of mental disorders. $5^{\text {th }}$ edition. Washington, D.C.: American Psychiatric Association; 2013.

[2] Centers for Disease Control and Prevention. Prevalence of autism spectrum disorders Autism and Developmental Disabilities Monitoring Network, 14 Sites, United States, 2002. Centers for Disease Control and Prevention. 2007; 56(1):12-28.

[3] Centers for Disease Control and Prevention. Prevalence of autism spectrum disorders: Autism and developmental disabilities monitoring network, United States, 2006. Centers for Disease Control and Prevention. 2009; 58(10):1-20.

[4] Centers for Disease Control and Prevention. Prevalence of autism spectrum disorders: Autism and developmental disabilities monitoring network, 14 sites, United States, 2008. Centers for Disease Control and Prevention. 2012; 61(3):1-19.

[5] Centers for Disease Control and Prevention. Prevalence of autism spectrum disorders among children aged 8 years: Autism and developmental disabilities monitoring network, 11 sites, United States, 2010. Centers for Disease Control and Prevention. 2014; 63(2):1-22.

[6] Bozorgnia A, Malekpour M, Abedi A. [Prevalence of autism in children 6 to 12 years old Shhrkrd 2009-2010 (Persian)]. Paper Presented at: The Regional Conference on Child and Adolescent Psychology, 26 May 2011, Kermanshah, Iran.

[7] Samadi SA, Mahmoodizadeh A, McConkey R. A national study of the prevalence of autism among five-year-old children in Iran. Autism. 2012; 16(1):5-14. doi: 10.1177/1362361311407091

[8] Samadi SA, McConkey R. Screening for autism in Iranian preschoolers: Contrasting M-CHAT and a scale developed in Iran. Journal of Autism and Developmental Disorders. 2015; 45(9):2908-16. doi: 10.1007/ s10803-015-2454-1

[9] Soto S, Linas K, Jacobstein D, Biel M, Migdal T, Anthony BJ. A review of cultural adaptations of screening tools for autism spectrum disorders. Autism. 2015; 19(6):646-61. doi: 10.1177/1362361314541012

[10] Howlin P, Asgharian A. The diagnosis of autism and Asperger syndrome: Findings from a survey of 770 families. Developmental Medicine \& Child Neurology. 1999; 41(12):834-9. doi: 10.1017/ s0012162299001656.

[11] Kamio Y, Inada N, Koyama T, Inokuchi E, Tsuchiya K, Kuroda M. Effectiveness of using the modified checklist for autism in toddlers in two-stage screening of autism spectrum disorder at the 18-month health check-up in Japan. Journal of Autism and Developmental Disorders. 2013; 44(1):194-203. doi: 10.1007/s10803-013-1864-1

[12] Herlihy L, Knoch K, Vibert B, Fein D. Parents' first concerns about toddlers with autism spectrum disorder: Effect of sibling status. Autism. 2015; 19(1):20-8. doi: 10.1177/1362361313509731

[13] Werner E, Dawson G, Osterling J, Dinno N. Brief report: Recognition of autism spectrum disorder before one year of age: A retrospective study based on home videotapes. Journal of autism and developmental disorders. 2000; 30(2):157-62. doi: 10.1023/a:1005463707029

[14] Charman T, Gotham K. Measurement issues: Screening and diagnostic instruments for autism spectrum disorders-lessons from research and practise. Child and Adolescent Mental Health. 2012; 18(1):52-63. doi: 10.1111/j.1475-3588.2012.00664.x

[15] Swinkels SHN, Dietz C, van Daalen E, Kerkhof IHGM, van Engeland $\mathrm{H}$, Buitelaar JK. Screening for Autistic spectrum in children aged 14 to 15 months. I: The development of the Early Screening of Autistic Traits Questionnaire (ESAT). Journal of Autism and Developmental Disorders. 2006; 36(6):723-32. doi: 10.1007/s10803-006-0115-0

[16] Dietz C, Swinkels S, van Daalen E, van Engeland H, Buitelaar JK. Screening for autistic spectrum disorder in children aged 14-15 Months. II: Population screening with the Early Screening of Autistic Traits Questionnaire (ESAT). Design and general findings. Journal of Autism and Developmental Disorders. 2006; 36(6):713-22. doi: 10.1007/ s10803-006-0114-1

[17] Oosterling IJ, Wensing M, Swinkels SH, van der Gaag RJ, Visser JC, Woudenberg $\mathrm{T}$, et al. Advancing early detection of autism spectrum disorder by applying an integrated two-stage screening approach. Journal of Child Psychology and Psychiatry. 2010; 51(3):250-8. doi: 10.1111/j.1469-7610.2009.02150.x

[18] Rogers SJ, Estes A, Lord C, Vismara L, Winter J, Fitzpatrick A, et al. Effects of a brief early start Denver model (ESDM)-based parent intervention on toddlers at risk for autism spectrum disorders: A randomized controlled trial. Journal of the American Academy of Child \& Adolescent Psychiatry. 2012; 51(10):1052-65. doi: 10.1016/j.jaac.2012.08.003

[19] Möricke E, Buitelaar JK, Rommelse NNJ. Do we need multiple informants when assessing autistic traits? The degree of report bias on offspring, self, and spouse ratings. Journal of Autism and Developmental Disorders. 2015; 46(1):164-75. doi: 10.1007/s10803-015-2562-y

[20] Möricke E, Swinkels SHN, Beuker KT, Buitelaar JK. Predictive value of subclinical autistic traits at age 14-15 months for behavioural and cognitive problems at age 3-5 years. European Child \& Adolescent Psychiatry. 2010; 19(8):659-68. doi: 10.1007/s00787-010-0103-y

[21] Oosterling I, Visser J, Swinkels S, Rommelse N, Donders R, Woudenberg $\mathrm{T}$, et al. Randomized controlled trial of the focus parent training for toddlers with autism: 1-year outcome. Journal of Autism and Developmental Disorders. 2010; 40(12):1447-58. doi: 10.1007/s10803-010$1004-0$

[22] Beuker KT, Schjølberg S, Lie KK, Swinkels S, Rommelse NNJ, Buitelaar JK. ESAT and M-CHAT as screening instruments for autism spectrum disorders at 18 months in the general population: Issues of overlap and association with clinical referrals. European Child \& Adolescent Psychiatry. 2014; 23(11):1081-91. doi: 10.1007/s00787-014-0561-8

[23] Inada N, Koyama T, Inokuchi E, Kuroda M, Kamio Y. Reliability and validity of the Japanese version of the Modified Checklist for Autism in Toddlers (M-CHAT). Research in Autism Spectrum Disorders. 2011; 5(1):330-6. doi: 10.1016/j.rasd.2010.04.016

[24] Ghamari-Givi H, Kian-Ersi F, Nasoudi R, Agh A, Mehrabadi S. [The comparison of effectiveness of applied behavioral analysis and treatment-education approach on stereotyped behavior, interactional and communicational problems in autistic children (Persian)]. Archives of Rehabilitation. 2012; 13(3):25-33.

[25] Nah YH, Young RL, Brewer N. Using the autism detection in early childhood (ADEC) and childhood autism rating scales (CARS) to predict long term outcomes in children with autism spectrum disorders. Journal of Autism and Developmental Disorders. 2014; 44(9):2301-10. doi: 10.1007/s10803-014-2102-1 
[26] Ahmadi S, Safari T, Hemmatian M, Khalili Z. [The psychometric properties of Gilliam Autism Rating Scale (GARS) (Persian)]. Journal of Cognitive and Behavioral Sciences. 2011; 1(1):87-104.

[27] Scarpa A, Reyes NM, Patriquin MA, Lorenzi J, Hassenfeldt TA, Desai VJ, et al. The modified checklist for autism in toddlers: Reliability in a diverse rural American sample. Journal of Autism and Developmental Disorders. 2013; 43(10):2269-79. doi: 10.1007/s10803-013-1779-x

[28] Nodehi Moghadam A, Niknahad A, Salavati M, Kazem-Nezhad A. [The adaptation and experience of four shoulder patient-centered outcome scores (Persian)]. Archives of Rehabilitation. 2012; 13(2):26-32.

[29] Chehelamirani N, Sahaf R, Rassafiani M, Bakhshi E. [Validity and reliability of WHOQOL-DIS Questionnaire in Iranian older people with disability (Persian)]. Archives of Rehabilitation. 2016; 16(4):334-345.

[30] Mohajer Rahbari M, Shariati M, Keramat A, Yunesian M, Eslami M, Mousavi SA, et al. Content validity of national post marriage educational program using mixed methods. Iranian Journal of Public Health. 2015; 44(4):535-542.

[31] Mayhew MJ, Powell JH. The development of a brief self-report questionnaire to measure "recent" Rash Impulsivity: A preliminary investigation of its validity and association with recent alcohol consumption. Addictive Behaviors. 2014; 39(11):1597-605. doi: 10.1016/j. addbeh.2014.03.022

[32] Trippolini MA, Dijkstra PU, Geertzen JHB, Reneman MF. Measurement properties of the spinal function sort in patients with sub-acute whiplash-associated disorders. Journal of Occupational Rehabilitation. 2015; 25(3):527-36. doi: 10.1007/s10926-014-9559-9

[33] Mahmoodi F, Zarifiyan T, Kazemi Y, SimaShirazi T. [Cultural adaptation and validation of the Persian version of the children's Communication Checklist-Cecond edition (CCC-2) (Persian)]. Journal of Research In Rehabilitation Sciences. 2014; 10(2):281-291.

[34] Polit DF, Beck CT, Owen SV. Is the CVI an acceptable indicator of content validity? Appraisal and recommendations. Research in Nursing and Health. 2007; 30(4):459-67. doi: 10.1002/nur.20199

[35] Hair Jr JF, Hult GTM, Ringle CM, Sarstedt M. A primer on Partial Least Squares Structural Equation Modeling (PLS-SEM). California: Sage Publications; 2013.

[36] Inada N, Koyama T, Inokuchi E, Kuroda M, Kamio Y. Reliability and validity of the Japanese version of the modified checklist for autism in toddlers (M-CHAT). Research in Autism Spectrum Disorders. 2011; 5(1):330-6. doi: 10.1016/j.rasd.2010.04.016

[37] Albores-Gallo L, Roldán-Ceballos O, Villarreal-Valdes G, BetanzosCruz BX, Santos-Sánchez C, Martínez-Jaime MM, et al. M-CHAT Mexican version validity and reliability and some cultural considerations. ISRN Neurology. 2012; 2012:1-7. doi: 10.5402/2012/408694

[38] Chung KM, Park S. Validity and reliability of the Korean Version of Autism Spectrum Disorders-Diagnostic for Children (ASD-DC). Research in Autism Spectrum Disorders. 2013; 7(12):1518-27. doi: 10.1016/j.rasd.2013.09.007

[39] Robins DL, Fein D, Barton ML, Green A. The modified checklist for autism in toddlers: An initial study investigating the early detection of autism and pervasive developmental disorders. Journal of Autism and Developmental Disorders. 2001; 31(2):131-144. doi: 10.1023/a:1010738829569 
